\title{
The Noncommutative Supersymmetric Nonlinear Sigma Model
}

\author{
H. O. Girotti ${ }^{a}$, M. Gomes ${ }^{b}$, V. O. Rivelles ${ }^{b}$ and A. J. da Silva ${ }^{b}$ \\ ${ }^{a}$ Instituto de Física, Universidade Federal do Rio Grande do Sul \\ Caixa Postal 15051, 91501-970 - Porto Alegre, RS, Brazil \\ E-mail: hgirotti@if.ufrgs.br \\ ${ }^{b}$ Instituto de Física, Universidade de São Paulo \\ Caixa Postal 66318, 05315-970, São Paulo - SP, Brazil \\ E-mail: mgomes, rivelles, ajsilva@fma.if.usp.br
}

\begin{abstract}
We show that the noncommutativity of space-time destroys the renormalizability of the $1 / N$ expansion of the $O(N)$ Gross-Neveu model. A similar statement holds for the noncommutative nonlinear sigma model. However, we show that, up to the subleading order in $1 / N$ expansion, the noncommutative supersymmetric $O(N)$ nonlinear sigma model becomes renormalizable in $D=3$. We also show that dynamical mass generation is restored and there is no catastrophic UV/IR mixing. Unlike the commutative case, we find that the Lagrange multiplier fields, which enforce the supersymmetric constraints, are also renormalized. For $D=2$ the divergence of the four point function of the basic scalar field, which in $D=3$ is absent, cannot be eliminated by means of a counterterm having the structure of a Moyal product.
\end{abstract}




\section{INTRODUCTION}

Recently, a great amount of interest has been devoted to the subject of noncommutative field theory. This interest has various origins as quantum gravity, string theory or just the investigations of the conceptual basis of field theory. It was found that, contrary to initial expectations, noncommutative models based on Moyal products are in general plagued with inconsistencies like the breaking of unitarity and causality [1] and the mixing of infrared/ultraviolet divergences [2-13]. Whenever possible, one could evade the problems of unitarity and causality by restricting the noncommutativity to the space coordinates only.

Noncommutative field theories are nonlocal field models where the nonlocality is a well defined consequence of the noncommutativity. For a given model it could happen that the ultraviolet divergences do not preserve the nonlocal Moyal structure of the bare vertices and the model turns out to be nonrenormalizable though it is renormalizable in ordinary space. After verifying renormalizability, one should yet determine up to what extent a noncommutative model retains the main features of its commutative counterpart. The answer to both questions depends on the details of the underlying interactions. For instance, it was recently shown that the $O(N)$ symmetry of the noncommutative linear sigma model can not be spontaneously broken for $N>2$, while, at least up to one loop, the same does not apply for the noncommutative $U(N)$ linear sigma model when the ordering of the quartic interaction is gauge invariant [14.

Most of the investigations on noncommutative field theories have been restricted to the first terms of the perturbative series. Extension to higher orders are hampered by technical difficulties and the UV/IR mixing. This last feature, namely, the entanglement of scales which mixes the ultraviolet and infrared behaviors leads to the breakdown of the perturbative scheme in many of the standard renormalizable theories. Fortunately, supersymmetric models [15] appear to be free from this drawback as it was shown for the Wess-Zumino model [16].

The question now is to decide whether the supersymmetric extension of a noncommutative field theory not only preserves the renormalizability but also other essential properties that its commutative counterpart may eventually possess.

In the present work we will use the $D$ dimensional $(2 \leq D<4) O(N)$ Gross-Neveu $(\mathrm{GN})$ model to illustrate these points. Although perturbatively renormalizable only for $D=2$, its commutative version is $1 / N$ expandable and exhibits dynamical mass generation for both $D=2$ and $D=3$ [17 20]. As we shall see, noncommutativity breaks both these aspects. Nevertheless, as in the Wess-Zumino model [16], supersymmetry allows to recover renormalizability and dynamical mass generation.

The supersymmetric partner of the GN model is the nonlinear sigma model whose noncommutative extension is afflicted with UV/IR mixing. As will be seen, supersymmetry also corrects this problem. It should be stressed, however, that, due to the nonlocal character of the Moyal product, the renormalization program for the noncommutative supersymmetric nonlinear sigma model presents new aspects, which are not shared by its commutative counterpart. In particular, as we shall prove, multiplicative renormalizations for the auxiliary fields become mandatory to achieve finite radiative corrections.

The paper is organized as follows. In section II we start by stressing some basic aspects of the $1 / N$ expansion of the commutative GN model which will prove crucial for our dis- 
cussion. Afterwards, we introduce its noncommutative version and explicitly demonstrate the breakdown of renormalizability. In this section we also discuss the UV/IR mixing in the $1 / N$ expansion of the nonlinear sigma model. In section III we pinpoint the tools needed to construct the $1 / N$ expansion of the supersymmetric nonlinear sigma model, which contains both the nonlinear sigma model and the GN model [22 27]. The noncommutative supersymmetric nonlinear sigma model is studied in section IV with special emphasis on the mechanism of mass generation. We also include in this section the computation of the leading corrections to the propagators of the basic fields. In section $\mathrm{V}$ we complete the renormalization program in $2+1$ dimensions, up to the subleading order in $1 / N$. Some remarks on the renormalization of the $1+1$ dimensional model and the conclusions are presented in section VI.

\section{THE GROSS-NEVEU AND THE NON LINEAR SIGMA MODELS}

The commutative $O(N)$ GN model is specified by the Lagrangian density

$$
\mathcal{L}=\frac{i}{2} \bar{\psi} \not \partial \psi+\frac{g}{4 N}(\bar{\psi} \psi)(\bar{\psi} \psi) .
$$

where $\psi_{i}, \quad i=1, \ldots N$ are two-component Majorana spinors. Only in two dimensions this model is perturbatively renormalizable. Although perturbatively nonrenormalizable in $2+1$ dimensions, the model is $1 / N$ expandable and presents some interesting aspects such as dynamical mass generation [18 20]. From now on our discussion will be restricted to $2 \leq D<4$.

To implement the $1 / N$ expansion one introduces an auxiliary field $\sigma$ which enables to write the Lagrangian of the theory as

$$
\mathcal{L}=\frac{i}{2} \bar{\psi} \not \partial \psi-\frac{\sigma}{2}(\bar{\psi} \psi)-\frac{N}{4 g} \sigma^{2}
$$

At the quantum level one should integrate over $\sigma$ which can develop a nonvanishing vacuum expectation value (VEV). We replace $\sigma$ by $\sigma+M$ where $M$ is the VEV of the original $\sigma$. The new Lagrangian is

$$
\mathcal{L}=\frac{i}{2} \bar{\psi} \not \partial \psi-\frac{M}{2} \bar{\psi} \psi-\frac{\sigma}{2}(\bar{\psi} \psi)-\frac{N}{4 g} \sigma^{2}-\frac{N}{2 g} M \sigma .
$$

Since, by construction, the new field $\sigma$ has zero VEV, the gap equation

$$
\frac{M}{2 g}-i \int \frac{d^{D} k}{(2 \pi)^{D}} \frac{M}{k^{2}-M^{2}}=0
$$

must be obeyed. After a Wick rotation one has

$$
\frac{M}{2 g}-\int \frac{d^{D} k}{(2 \pi)^{D}} \frac{M}{k_{E}^{2}+M^{2}}=0 .
$$

The ultraviolet divergence in the above integral may be eliminated by means of a coupling constant renormalization. Indeed, by defining the renormalized coupling constant through 


$$
\frac{1}{g}=\frac{1}{g_{R}}+2 \int \frac{d^{D} k}{(2 \pi)^{D}} \frac{1}{k_{E}^{2}+\mu^{2}}
$$

the divergence is canceled. In $2+1$ dimension one finds

$$
\frac{1}{g_{R}}=\frac{\mu-|M|}{2 \pi}
$$

and therefore only for $-\frac{1}{g_{R}}+\frac{\mu}{2 \pi}>0$ it is possible to have $M \neq 0$. Otherwise $M$ is necessarily zero. In two dimensions no such restriction exists. Whatever the case, we shall only focus on the massive phase.

We shall next compute the propagator for the auxiliary field. It is given by $-1 / F(p)$, where

$$
F(p)=-\frac{i N}{2 g}-N \int \frac{d^{D} k}{(2 \pi)^{D}} \frac{k \cdot(k+p)+M^{2}}{\left(k^{2}-M^{2}\right)\left[(k+p)^{2}-M^{2}\right]}
$$

is the two point function of the sigma field. This last integral is divergent but after taking into account (2.4) it becomes

$$
F(p)=\frac{\left(p^{2}-4 M^{2}\right) N}{2} \int \frac{d^{D} k}{(2 \pi)^{D}} \frac{1}{\left(k^{2}-M^{2}\right)\left[(k+p)^{2}-M^{2}\right]},
$$

which is finite for $D<4$. We see that the above cancellation of divergences results from a fine tuning between the divergence in the integral in (2.8) and the one in the gap equation.

Let us turn our attention to the noncommutative version of the model. By introducing Moyal products we arrive at

$$
S_{G N}=\int d^{D} x\left[\frac{i}{2} \bar{\psi} \not \partial \psi-\frac{M}{2} \bar{\psi} \psi-\frac{1}{2} \sigma \star(\bar{\psi} \star \psi)-\frac{N}{4 g} \sigma^{2}-\frac{N}{2 g} M \sigma\right] .
$$

As can be checked, the path integration on $\sigma$ leads to a noncommutative version of (2.1) in which the four-fermion interaction is $\bar{\psi}_{i} \star \psi_{i} \star \bar{\psi}_{j} \star \psi_{j}$. A more general $O(N)$ noncommutative four-fermion interaction may involve the term $\bar{\psi}_{i} \star \bar{\psi}_{j} \star \psi_{i} \star \psi_{j}$. However this last combination does not have a simple $1 / N$ expansion and it will not be considered in this work.

Since the Moyal product does not affect the quadratic part, the propagator for $\psi$ is as before. On the other hand, in momentum space the trilinear vertex has to be multiplied by $\cos \left(p_{1} \wedge p_{2}\right)$ where $p_{1}$ and $p_{2}$ are the momenta through the fermion lines 1 . Then, the gap equation is not modified whereas, for the proper two point function of the $\sigma$ field, one obtains

$$
\mathcal{F}(p)=-\frac{i N}{2 g}-N \int \frac{d^{D} k}{(2 \pi)^{D}} \cos ^{2}(k \wedge p) \frac{k \cdot(k+p)+M^{2}}{\left(k^{2}-M^{2}\right)\left[(k+p)^{2}-M^{2}\right]}
$$

${ }^{1}$ Here we have introduced the notation $a \wedge b=1 / 2 a^{\mu} b^{\nu} \Theta_{\mu \nu}$, where $\Theta_{\mu \nu}$ is the anti-symmetric constant matrix characterizing the noncommutativity of the underlying space. 
and we see that the divergent parts do not match anymore. The model is no longer renormalizable. A similar conclusion was arrived in [21].

An analogous situation arises in connection with the Goldstone theorem within the context of the $O(N)$ linear sigma model. Indeed, the pion propagator counterterm, which is determined by the vanishing of the VEV of the sigma field, is not modified by the noncommutativity. However, for $N>2$, the graphs contributing to the one loop corrections of the pion propagator are altered thus destroying the renormalizability of the model [14].

As a side observation, notice that, up to the leading order of $1 / N$, no inconsistency would arise had we employed Dirac instead of Majorana spinors. This is so because the oscillating exponentials, characteristics of the noncommutativity, cancel in the leading order contributions to the self-energy parts. The same does not hold at higher orders of $1 / N$.

We would like to remark that the noncommutative version of the nonlinear sigma model, which happens to be the supersymmetric partner of the GN model, also presents inconsistencies. The model is specified by the Lagrangian

$$
\mathcal{L}=-\frac{1}{2} \varphi\left(\partial^{2}+M^{2}\right) \varphi+\frac{1}{2} \lambda \star \varphi \star \varphi-\frac{N}{2 g} \lambda,
$$

where $\varphi_{i}, i=1, \ldots, N$ are real scalar fields, $M$ is, as before, the generated mass and $\lambda$ is an auxiliary field which implements the nonlinear constraint $\varphi \star \varphi=N / g$. The leading correction to the self-energy of the $\varphi$ field, shown in Fig. 1 $a$, is

$$
-i \int \frac{d^{2} k}{(2 \pi)^{2}} \frac{\cos ^{2}(k \wedge p)}{(k+p)^{2}-M^{2}} \Delta_{\lambda}(k),
$$

where $\Delta_{\lambda}$ is the propagator for the $\lambda$ field. This expression may be decomposed into a sum of a planar (quadratically divergent) piece and a nonplanar one. The nonplanar contribution is ultraviolet finite but diverges quadratically for small momenta (UV/IR mixing [2]) thus destroying the $1 / N$ expansion of the model. Moreover, an attempt to solve this difficulty by generalizing the definition of 1PI diagram [24] amounts to add the contribution from graph in Fig. $1 b$ which is given by

$$
-i \Delta_{\lambda}(0) \int \frac{d^{2} k}{(2 \pi)^{2}} \frac{d^{2} q}{(2 \pi)^{2}} \frac{\cos ^{2}(k \wedge q)}{(k+q)^{2}-M^{2}} \frac{1}{\left(q^{2}-M^{2}\right)^{2}} \Delta_{\lambda}(k) .
$$

Like in the commutative case, the $\lambda$ field propagator $\Delta_{\lambda}(k)$ behaves as $k^{2}$ when $k \rightarrow \infty$. Then, for small $p$ the integral (2.13) gives origin to a quadratic infrared divergence which cannot be compensated by (2.14). This is the UV/IR mixing which, as already mentioned, breaks the $1 / N$ expansion.

Before demonstrating that the noncommutative supersymmetric nonlinear sigma model remains renormalizable, we shall recall some aspects of its commutative counterpart [22 27].

\section{THE SUPERSYMMETRIC NONLINEAR SIGMA MODEL}

The commutative supersymmetric nonlinear sigma model is described by the Lagrangian density 


$$
\mathcal{L}=-\frac{1}{2} \varphi \partial^{2} \varphi+\frac{i}{2} \bar{\psi} \not \partial \psi+\frac{1}{2} F^{2}+\sigma \varphi_{j} F_{j}+\frac{1}{2} \lambda \varphi^{2}-\frac{1}{2} \sigma \bar{\psi} \psi-\bar{\xi} \psi_{j} \varphi_{j}-\frac{N}{2 g} \lambda,
$$

where $\varphi_{i}, \psi_{i}$ and $F_{i}, i=1, \ldots, N$, are, respectively, real scalar, two-component Majorana spinor and auxiliary scalar fields. Furthermore, $\sigma, \lambda$ and $\xi$ are Lagrange multipliers which implement the supersymmetric constraints

$$
\begin{aligned}
\varphi_{i} \varphi_{i} & =\frac{N}{g}, \\
\varphi_{i} \psi_{i} & =0, \\
\varphi_{i} F_{i} & =\frac{1}{2} \bar{\psi} \psi .
\end{aligned}
$$

A more symmetric form for (3.1) is obtained after the linear change of variables $\lambda \rightarrow \lambda+2 M \sigma$, $F \rightarrow F-M \varphi$ where $M=\langle\sigma\rangle$. Afterwards, one performs the shifts $\sigma \rightarrow \sigma+M$ and $\lambda \rightarrow \lambda+\lambda_{0}$, where $\lambda_{0}=\langle\lambda\rangle$, yielding

$$
\begin{aligned}
\mathcal{L}= & -\frac{1}{2} \varphi\left(\partial^{2}+M^{2}\right) \varphi+\frac{1}{2} \bar{\psi}(i \not \partial-M) \psi+\frac{1}{2} F^{2}+M^{2} \varphi^{2}+\frac{1}{2} \lambda_{0} \varphi^{2} \\
& +\frac{1}{2} \lambda \varphi^{2}+\sigma \varphi_{j} F_{j}-\frac{1}{2} \sigma \bar{\psi} \psi-\bar{\xi} \psi_{j} \varphi_{j}-\frac{N}{2 g} \lambda-\frac{N}{g} M \sigma .
\end{aligned}
$$

Supersymmetry demands $\lambda_{0}=-2 M^{2}$ and the gap equations arising from $\langle\lambda\rangle=\langle\sigma\rangle=0$, for $M \neq 0$, are found to imply

$$
\int \frac{d^{D} k}{(2 \pi)^{D}} \frac{i}{k^{2}-M^{2}}=\frac{1}{g}
$$

so that as for the GN model an infinite coupling constant renormalization is required. We must again investigate up to what extent such normalization affects the computation of the propagator for the sigma field. To that end we list the propagators for the basic fields $\varphi, \psi$ and for the auxiliary field $F$. They are

$$
\begin{aligned}
& \Delta_{\varphi_{i} \varphi_{j}}(p)=\Delta_{i j}=\frac{i \delta_{i j}}{p^{2}-M^{2}}, \\
& S_{F}(p)=\frac{i \delta_{i j}}{\not p-M}, \\
& \Delta_{F_{i} F_{j}}=i \delta_{i j} .
\end{aligned}
$$

Unlike the GN model, the finiteness of the $\sigma$ field propagator does not depend on the renormalization of the coupling constant $g$. In fact, the proper part $F_{\sigma}$ of the two point function of the sigma field receives contributions from the second and third terms in the second line of (3.5). One finds that

$$
F_{\sigma}(p)=N \int \frac{d^{D} k}{(2 \pi)^{D}} \frac{1}{k^{2}-M^{2}}-N \int \frac{d^{D} k}{(2 \pi)^{D}} \frac{k \cdot(k+p)+M^{2}}{\left[(p+k)^{2}-M^{2}\right]\left[k^{2}-M^{2}\right]},
$$


where the first term arises from the second order contribution of the $\sigma \varphi F$ vertex and the last one originates from the second order contribution of the $\sigma \bar{\psi} \psi$ vertex. After a straightforward algebra one arrives at

$$
F_{\sigma}(p)=\frac{\left(p^{2}-4 M^{2}\right) N}{2} \int \frac{d^{D} k}{(2 \pi)^{D}} \frac{1}{\left(k^{2}-M^{2}\right)\left[(k+p)^{2}-M^{2}\right]},
$$

which is identical to (2.9). We strongly remark that, in contradistinction to (2.9), the finiteness here is not a consequence of a gap equation.

\section{THE NONCOMMUTATIVE SUPERSYMMETRIC NONLINEAR SIGMA MODEL: RENORMALIZATION OF THE TWO POINT FUNCTIONS}

The noncommutative supersymmetric nonlinear sigma model is specified by

$$
\begin{aligned}
S= & \int\left\{-\frac{1}{2} \varphi\left(\partial^{2}+M^{2}\right) \varphi+\frac{1}{2} \bar{\psi}(i \not \partial-M) \psi+\frac{1}{2} F^{2}+\frac{\lambda}{2} \star \varphi \star \varphi\right. \\
& -\frac{1}{2} F_{j} \star\left(\sigma \star \varphi_{j}+\varphi_{j} \star \sigma\right)-\frac{1}{2} \sigma \star \bar{\psi} \star \psi-\frac{1}{2}(\bar{\xi} \star \psi \star \varphi+\bar{\xi} \star \varphi \star \psi) \\
& \left.-\frac{N}{2 g} \lambda-\frac{N M \sigma}{g}\right\} d^{D} x .
\end{aligned}
$$

It should be noticed that the symmetrized forms used above are the only noncommutative supersymmetric extensions for the terms $\sigma \varphi F$ and $\bar{\xi} \psi \varphi$. It must also be emphasized that the noncommutative supersymmetry transformations are identical to the commutative ones since they are linear in the fields and no Moyal products are, therefore, involved.

Since the quadratic part of the action was not modified, the free propagators of the basic fields $\varphi, \psi$ and $F$ remain unaltered. As for the vertices, they acquire cosine factors as follows

$$
\begin{array}{cccc}
\lambda \varphi^{2} & \text { vertex: } & \frac{i}{2} \cos \left(p_{1} \wedge p_{2}\right), \\
\sigma \varphi F & \text { vertex: } & -i \cos \left(p_{1} \wedge p_{2}\right), \\
\bar{\psi} \psi \sigma & \text { vertex: } & -\frac{i}{2} \cos \left(p_{1} \wedge p_{2}\right) \\
\bar{\xi} \psi \varphi & \text { vertex: } & -i \cos \left(p_{1} \wedge p_{2}\right) .
\end{array}
$$

Using the above rules one can compute the leading order propagators for the Lagrange multiplier fields. For the proper part of the $\sigma$ field, $\mathcal{F}_{\sigma}$, one merely obtains the expression (3.8) modified by the presence of the factor $\cos ^{2}(k \wedge p)$ in each integral. Hence,

$$
\mathcal{F}_{\sigma}(p)=\frac{\left(p^{2}-4 M^{2}\right) N}{2} \int \frac{d^{D} k}{(2 \pi)^{D}} \frac{\cos ^{2}(k \wedge p)}{\left(k^{2}-M^{2}\right)\left[(k+p)^{2}-M^{2}\right]},
$$

which is well behaved in both the infrared and ultraviolet regions. The propagator for the $\sigma$ field is, of course, $\Delta_{\sigma}=-1 / \mathcal{F}_{\sigma}$.

The expressions for the $\lambda$ and $\xi$ propagators are given by $\Delta_{\lambda}=-1 / \mathcal{F}_{\lambda}$ and $S_{\xi}=-1 / \mathcal{F}_{\xi}$, respectively, where 


$$
\mathcal{F}_{\lambda}(p)=\frac{N}{2} \int \frac{d^{D} k}{(2 \pi)^{D}} \cos ^{2}(k \wedge p) \frac{1}{\left[(k+p)^{2}-M^{2}\right]\left[k^{2}-M^{2}\right]}
$$

and

$$
\begin{aligned}
& \mathcal{F}_{\xi}(p)=N \int \frac{d^{D} k}{(2 \pi)^{D}} \cos ^{2}(k \wedge p) \frac{-\not k+M}{\left[(k+p)^{2}-M^{2}\right]\left[k^{2}-M^{2}\right]} \\
& =N \frac{(\not p+2 M)}{2} \int \frac{d^{D} k}{(2 \pi)^{D}} \cos ^{2}(k \wedge p) \frac{1}{\left[(k+p)^{2}-M^{2}\right]\left[k^{2}-M^{2}\right]},
\end{aligned}
$$

which are ultraviolet finite and without singularities for small $p$. We observe that all the Lagrange multiplier field propagators are of order $1 / N$.

Our graphical notation for the propagators is presented in Fig. 2. Observe that, being a constant, the $F$ propagator could be omitted altogether, but one is not to forget the cosine factors in the original graph.

Due to the oscillating nature of the cosines some of the integrals constructed with the above rules will be finite but in general divergences will survive. The degree of superficial divergence for a generic 1PI graph $\gamma$ is

$$
d(\gamma)=D-\frac{(D-1)}{2} N_{\psi}-\frac{(D-2)}{2} N_{\varphi}-\frac{D}{2} N_{F}-N_{\sigma}-\frac{3}{2} N_{\xi}-2 N_{\lambda}
$$

where $N_{\mathcal{O}}$ is the number of external lines associated to the field $\mathcal{O}$. Potentially dangerous diagrams are those contributing to the self-energies of the $\varphi$ and $\psi$ fields since, in principle, they are quadratic and linearly divergent, respectively.

In lowest order there are three graphs contributing to the $\varphi$ field self-energy which are shown in Fig. 3 . In a self explanatory notation, the analytic expressions associated with them are

$$
\begin{gathered}
\Sigma_{\varphi}^{a}=-i \int \frac{d^{D} k}{(2 \pi)^{D}} \frac{\cos ^{2}(k \wedge p)}{(k+p)^{2}-M^{2}} \Delta_{\lambda}(k), \\
\sum_{\varphi}^{b}=i \int \frac{d^{D} k}{(2 \pi)^{D}} \operatorname{Tr}\left\{\frac{\cos ^{2}(k \wedge p)}{(\not \gamma+\not h)-M} \frac{1}{\not \gamma+2 M}\right\} \Delta_{\lambda}(k),
\end{gathered}
$$

and

$$
\Sigma_{\varphi}^{c}=-i \int \frac{d^{D} k}{(2 \pi)^{D}} \frac{\cos ^{2}(k \wedge p)}{k^{2}-4 M^{2}} \Delta_{\lambda}(k)
$$

By adding these three expressions we get

$$
\Sigma_{\varphi}=-i\left(p^{2}-M^{2}\right) \int \frac{d^{D} k}{(2 \pi)^{D}} \frac{\cos ^{2}(k \wedge p)}{\left[(k+p)^{2}-M^{2}\right]\left[k^{2}-4 M^{2}\right]} \Delta_{\lambda}(k) .
$$

Individually each of the graphs in Fig. 3 is quadratically divergent but their sum diverges only logarithmically. This divergence can be eliminated by a wave function renormalization of the $\varphi$ field. Notice that, in spite of the presence of $\cos ^{2}(k \wedge p)$, the divergent part of (4.10) 
coincides with the corresponding one in the commutative case. Similar result holds for the self-energy of the $\psi$ field. Indeed, from Fig. 4 we obtain

$$
\Sigma_{\psi}^{a}=-i \int \frac{d^{D} k}{(2 \pi)^{D}} \frac{(k+\not p+M) \cos ^{2}(k \wedge p)}{\left[(k+p)^{2}-M^{2}\right]\left[k^{2}-4 M^{2}\right]} \Delta_{\lambda}(k)
$$

and

$$
\Sigma_{\psi}^{b}=i \int \frac{d^{D} k}{(2 \pi)^{D}} \frac{(k /+2 M) \cos ^{2}(k \wedge p)}{\left[(k+p)^{2}-M^{2}\right]\left[k^{2}-4 M^{2}\right]} \Delta_{\lambda}(k)
$$

so that

$$
\Sigma_{\psi}=-i(\not \prime-M) \int \frac{d^{D} k}{(2 \pi)^{D}} \frac{\cos ^{2}(k \wedge p)}{\left[(k+p)^{2}-M^{2}\right]\left[k^{2}-4 M^{2}\right]} \Delta_{\lambda}(k) .
$$

We see that the leading divergence is again canceled and just remains a logarithmic one which may be absorbed by a wave function renormalization of the $\psi$ field. A similar analysis of the logarithimic divergence present in the $F$ field propagator reveals that it can be also removed by a wave function renormalization of the $F$ field.

We stress that the renormalization factors for the fields $\varphi, \psi$ and $F$ are the same. Thus, up to this point, the renormalization of the noncommutative model preserves supersymmetry.

\section{RENORMALIZATION OF THE $N$ POINT FUNCTIONS}

To complete our study of the renormalization program we focus next on the four point function of the scalar field $\varphi, \Gamma_{i_{1} i_{2} i_{3} i_{4}}^{(4)}\left(p_{1}, p_{2}, p_{3}, p_{4}\right)$, where the subscripts are $O(N)$ indices. Without loosing generality we choose $i_{1}=i_{3}$ and $i_{2}=i_{4}$ but $i_{1} \neq i_{2}$. The diagrams are those in Fig. 5 whose associated amplitudes are

$$
\begin{aligned}
\Gamma_{a}^{(4)} & =\int \frac{d^{D} k}{(2 \pi)^{D}} \frac{i}{\left(k+p_{1}\right)^{2}-M^{2}} \frac{i}{\left(-k+p_{2}\right)^{2}-M^{2}} \Delta_{\lambda}(k) \Delta_{\lambda}\left(k+p_{1}-p_{3}\right) \mathcal{C}(k), \\
\Gamma_{b}^{(4)} & =\int \frac{d^{D} k}{(2 \pi)^{D}} i^{2} \Delta_{\sigma}(k) \Delta_{\sigma}\left(k+p_{1}-p_{3}\right) \mathcal{C}(k), \\
\Gamma_{c}^{(4)} & =-\int \frac{d^{D} k}{(2 \pi)^{D}} \operatorname{Tr}\left[\frac{i}{\left(k /+\not p_{1}\right)-M} S_{\xi}\left(k+p_{1}-p_{3}\right) \frac{i}{\left(k /-p_{2}\right)-M} S_{\xi}(k)\right] \mathcal{C}(k),
\end{aligned}
$$

where

$$
\mathcal{C}(k) \equiv \cos \left(k \wedge p_{1}\right) \cos \left(k \wedge p_{2}\right) \cos \left[\left(k+p_{1}\right) \wedge p_{3}\right] \cos \left[\left(k-p_{2}\right) \wedge p_{4}\right]
$$

In $D=3$ the above integrals are linearly divergent. However, as far as the sum of these amplitudes is concerned, it is readily seen that the leading divergence cancels and that the subleading one vanishes under symmetric integration. These considerations hold irrespective of the presence of the cosine factors in the function $\mathcal{C}(k)$. Thus, there is no UV/IR mixing that would eventually give rise to infrared divergences.

For $D=2$ each integral in (5.1) is quadratically divergent and in their sum a logarithmic divergence still survives. An explicit calculation shows that this divergence cannot be 
removed by a counterterm whose nonlocal structure arises from a combination of Moyal products of $\varphi$ fields. Because of this, in the remaining of this section, we restrict our discussion to $D=3$. Further remarks on $D=2$ will be postponed to the conclusions.

The graphs contributing to the six point vertex functions of the $\varphi$ field are displayed in Fig. 6. Since each graph in the set has the same number of vertices, the cosine factor is the same for all of them and, therefore, the cancellation among the divergent parts proceeds as in the commutative case.

As for the three point function, $\Gamma_{\lambda \varphi \varphi}$, depicted in Fig. [1, we notice that the diagrams can be grouped into two sets according to the number of cosine factors. Indeed, diagram $7 a$ exhibits three cosine factors while diagrams $7 b, 7 c$ and $7 d$ each contains five cosine factors. We have

$$
\Gamma_{a}^{(3)}\left(p_{1}, p_{2}\right)=\int \frac{d^{3} k}{(2 \pi)^{3}} I_{a}\left(k, p_{1}, p_{2}\right) \mathcal{C}_{3}
$$

and

$$
\Gamma_{b+c+d}^{(3)}\left(p_{1}, p_{2}\right)=\int \frac{d^{3} k}{(2 \pi)^{3}} \frac{d^{3} q}{(2 \pi)^{3}} I_{b+c+d}\left(k, q, p_{1}, p_{2}\right) \mathcal{C}_{5}
$$

where $I_{a}$ and $I_{b+c+d}$ are the same integrands as in the commutative case, and $\mathcal{C}_{3}$ and $\mathcal{C}_{5}$ are the cosine factors

$$
\begin{aligned}
\mathcal{C}_{3}= & \cos \left(k \wedge p_{1}\right) \cos \left(k \wedge p_{2}\right) \cos \left(k \wedge p_{1}+k \wedge p_{2}+p_{1} \wedge p_{2}\right)=\frac{1}{4} \cos \left(p_{1} \wedge p_{2}\right)+\ldots \\
\mathcal{C}_{5}= & \cos \left(q \wedge p_{1}\right) \cos \left(q \wedge p_{2}\right) \cos \left(k \wedge p_{1}+k \wedge p_{2}+p_{1} \wedge p_{2}\right) \cos \left(q \wedge p_{1}-k \wedge p_{1}+q \wedge k\right) \\
& \cos \left(k \wedge p_{2}-q \wedge p_{2}+q \wedge k\right)=\frac{1}{16} \cos \left(p_{1} \wedge p_{2}\right)+\ldots
\end{aligned}
$$

where the ellipsis indicate terms which give finite nonplanar contributions to the corresponding Feynman integrals. Hence, the divergent part of each set of graphs becomes altered with respect to the commutative regime [27] in such a way that it can no longer be absorbed by just a wave function renormalization of the $\varphi$ field. A wave function renormalization of the auxiliary field $\lambda$ is now needed. The same applies to the three point functions $\Gamma_{\sigma \bar{\psi} \psi}$ and $\Gamma_{\bar{\psi} \xi \varphi}$. Supersymmetry requires that the renormalization of $\sigma, \xi$ and $\lambda$ be the same, which can be verified to be case. This is to be contrasted with the situation in the commutative setting where no renormalization is required for the Lagrange multiplier fields.

\section{FINAL REMARKS AND CONCLUSIONS}

In the commutative GN model the coupling constant plays a dual role, eliminating divergences in both, the gap equation and the proper two point function of the Lagrange multiplier field $\sigma$. Due to the presence of the cosine factors, this property is no longer valid at the noncommutative level and the $O(N)$ model becomes nonrenormalizable. The noncommutative nonlinear sigma model is afflicted by a different type of inconsistency, namely the UV/IR mixing which destroys its $1 / N$ expansion. However, supersymmetry blends these

models together and, for $D=3$, leaves us with a consistent noncommutative quantum field 
theory. It is worth mentioning that the noncommutative $U(N)$ GN model by itself does not suffer from the above inconsistencies in the leading order of $1 / N$. Nevertheless, this does not apply to higher orders.

In $D=2$ the noncommutative supersymmetric nolinear model possesses certain peculiarities which are not present in $D=3$. Since the scalar field in $D=2$ is dimensionless, any graph involving an arbitrary number of external $\varphi$ lines is quadratically divergent. As exemplified by the four point function $\Gamma_{i_{1} i_{2} i_{3} i_{4}}^{(4)}\left(p_{1}, p_{2}, p_{3}, p_{4}\right)$ of the $\varphi$ field, supersymmetry provides a partial cancellation of divergences but a logarithmic one remains. The counterterm needed to remove such divergence would have the form

$$
\int \prod_{i=1}^{4} \frac{d^{2} k_{i}}{(2 \pi)^{2}} \cos \left[k_{1} \wedge k_{2}-k_{3} \wedge k_{4}\right](2 \pi)^{2} \delta\left(k_{1}+k_{2}+k_{3}+k_{4}\right) \tilde{\varphi}_{i}\left(k_{1}\right) \tilde{\varphi}_{j}\left(k_{2}\right) \tilde{\varphi}_{i}\left(k_{3}\right) \tilde{\varphi}_{j}\left(k_{4}\right),
$$

where $\tilde{\varphi}_{l}(k)$ is the Fourier transform of $\varphi(x)$. However, (6.1) can not be written in terms of the two independent Moyal orderings $\int d^{2} x \varphi_{i} \star \varphi_{i} \star \varphi_{j} \star \varphi_{j}$ and $\int d^{2} x \varphi_{i} \star \varphi_{j} \star \varphi_{i} \star \varphi_{j}$. Alternatively, one may entertain the possibility of canceling the divergences by generalizing the definition of 1PI diagram as suggested in [24] for the commutative nonlinear sigma model. Nevertheless, the unbalance of cosine factors precludes this mechanism. These observations cast doubts on the renormalizability of the noncommutative supersymmetric $O(N)$ nonlinear sigma model in two space-time dimensions.

As remarked before, the formulation of the noncommutative four-fermion interaction allows for a term of the form $\bar{\psi}_{i} \star \bar{\psi}_{j} \star \psi_{i} \star \psi_{j}$. This is an interesting problem since the $1 / N$ expansion involves now an auxiliary tensor field. In this connection, the $1 / N$ expansion for matrix models in comutative space has been discussed in the literature [28]

Summarizing, in this paper and in a previous one we have verified that supersymmetrization corrects some of the difficulties arising in the formulation of a noncommutative model as, for example the UV/IR mixing. We would like to mention that another possibility for solving the UV/IR problem, based on a resummation of the perturbative series, has been considered in the literature [29].

\section{ACKNOWLEDGMENTS}

This work was partially supported by Fundação de Amparo à Pesquisa do Estado de São Paulo (FAPESP) and Conselho Nacional de Desenvolvimento Científico e Tecnológico (CNPq). H.O.G and V.O.R also acknowledge support from PRONEX under contract CNPq $66.2002 / 1998-99$. 


\section{REFERENCES}

[1] N. Seiberg, L. Susskind and N. Toumbas, "Space/Time Non-Commutativity and Causality", JHEP 0006, 044 (2000), hep-th/0005015; J. Gomis and T. Mehen, "SpaceTime Noncommutative Field Theories and Unitarity", hep-th/0005129.

[2] S. Minwalla, M. V. Raamsdonk, N. Seiberg, "Noncommutative Perturbative Dynamics", JHEP 0002, 020 (2000), hep-th/9912072; "Comments on Noncommutative Perturbative Dynamics", hep-th/0002186.

[3] A. Matusis, L. Susskind, N. Toumba, "The IR/UV connection in the noncommutative gauge theories", hep-th/0002075.

[4] M. Hayakawa, "Perturbative Analysis on Infrared Aspects of Noncommutative QED on $R^{4}$ ", Phys. Lett. B478 (2000) 394, hep-th/9912094; "Perturbative Analysis on Infrared and Ultraviolet Aspects of Noncommutative QED on $R^{4}$ ", hep-th/9912167.

[5] I. Ya. Aref'eva, D. M. Belov, A. S. Koshelev and O. A. Rytchkov, "Renormalizability and UV/IR mixing in Noncommutative Theories with Scalar Fields", Phys. Lett. B487 (2000) 357.

[6] I. Ya. Aref'eva, D. M. Belov and A. S. Koshelev, "A Note on UV/IR for Noncommutative Complex Scalar Field", hep-th/0001215

[7] C. P. Martin and D. Sanchez-Ruiz, "The One-loop UV Divergent Structure of U(1) Yang-Mills Theory on Noncommutative $R^{4}$ ", Phys. Rev. Lett. 83 (1999) 476, hepth/9903077.

[8] H. Grosse, T. Krajewski and R. Wulkenhaar, "Renormalization of Noncommutative Yang-Mills Theories: A Simple Example", hep-th/0001182.

[9] A. Armoni, "Perturbative Dynamics of Noncommutative Yang-Mills Field Theory", hep-th/0005208

[10] L. Bonora, M. Schnabl and A. Tomasiello, "A note on consistent anomalies in noncommutative YM theories", Phys. Lett. B485 (2000) 311, hep-th/0002210.

[11] M. M. Sheikh-Jabbari, "Renormalizability of the Supersymmetric Yang-Mills Theories on the Noncommutative Torus", hep-th/9903107, JHEP 9906, 015 (1999).

[12] I. Chepelev and R. Roiban, "Renormalization of Quantum field Theories on Noncommutative $R^{d}$. I: Scalars," hep-th/9911098.

[13] J. M. Gracia-Bondia and C. P. Martin, "Chiral gauge Anomalies on Noncommutative $R^{4}, "$ hep-th/0002171.

[14] B. A. Campbell and K. Kaminsky, "Noncommutative Field Theory and Spontaneous Symmetry Breaking", Nucl. Phys. B581 (2000) 240, hep-th/0003137; "Noncommutative Linear Sigma Models", hep-th/0102022; F. J. Petriello, "The Higgs Mechanism in Noncommutative Gauge Theories", hep-th/0101109.

[15] C. Chu and F. Zamora, "Manifest Supersymmetry in Non-Commutative Geometry," hep-th/9912153; S. Ferrara and M. A. Lledo, "Some aspects of Deformations of Supersymmetric Field Theories," hep-th/0002084; S. Terashima, "A Note on Superfields and Noncommutative Geometry," hep- th/0002119.

[16] H. O. Girotti, M. Gomes, V. O. Rivelles and A. J. da Silva, "A Consistent Noncommutative Field Theory: The Wess-Zumino Model", Nucl. Phys. B587 (2000) 299, hepth/0005272; "The Low Energy Limit of the Noncommutative Wess-Zumino Model", hep-th/0101159.

[17] D. Gross and A. Neveu, Phys. Rev. D10 (1974) 3235. 
[18] D. Gross, Les Houches 1975, Methods in Field Theory, eds. R. Balian and J. Zinn-Justin, North-Holland, Amsterdam, 1976.

[19] G. Gat, A. Kovner and B. Rosenstein, Nucl. Phys. B385 (1992) 76

[20] M. Gomes, V. O Rivelles and A. J. da Silva, Phys. Rev. D41 (1990) 1363.

[21] E. T. Akhmedov, P. DeBoer and G. W. Semenoff, "Running Couplings and Triviality of Field Theories on Noncommutative Spaces", hep-th/0010003; JHEP 0106, 009 (2001).

[22] O. Alvarez, Phys. Rev. D 17 (1978) 1123.

[23] E. Witten, "Supersymmetric Form of the Nonlinear $\sigma$ Model in Two Dimensions", Phys. Rev. D 16 (1977) 2991.

[24] I. Ya. Aref'eva, Theor. Math. Phys. 36 (1979) 573; Ann. Phys. (NY) 117 (1979) 393; I. Ya. Aref'eva, E. R. Nissimov and S. J. Pacheva, Commun. Math. Phys. 71 (1980) 213. See also J. H. Lowenstein and E. R. Speer, Nucl.. Phys. B158 (1979) 397.

[25] A. D'Adda, P. Di Vecchia and M. Luscher, Nucl. Phys. B 152 (1979) 125.

[26] A. C. Davis, J. A. Gracey, A. J. Macfarlane and M. G. Mithcard, Nucl. Phys. B 314 (1989) 439.

[27] V. G. Koures and K. T. Mahanthappa, Phys. Rev. D 43 (1991) 3428.

[28] G. 't Hooft, Nucl. Phys. B72 (1974) 461.

[29] L. Griguolo and M. Pietroni, "Hard Noncommutative loops Resummation", hepth/0102070; JHEP 0105, 032 (2001). 


\section{FIGURES}
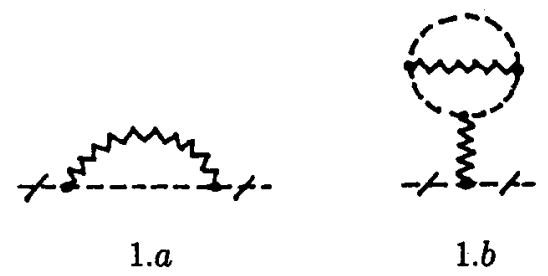

FIG. 1. Order $1 / N$ contributions to the self-energy of the $\varphi$ field. Dashed and wavy lines represent, respectively, the propagators of $\varphi$ and $\lambda$ fields.

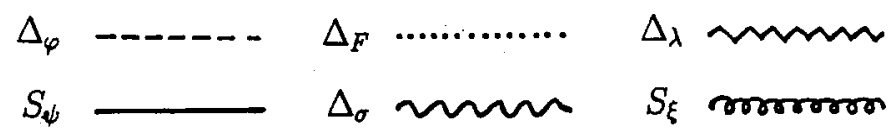

FIG. 2. Graphical representation for the propagators.

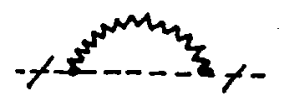

3. $a$

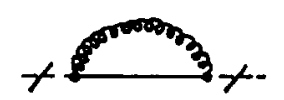

$3 . b$

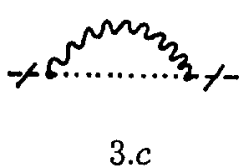

FIG. 3. $1 / N$ corrections to the $\varphi$ field self-energy. 


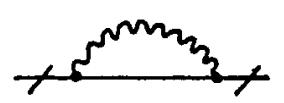

4. $a$

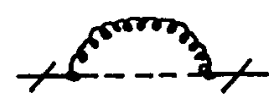

4.b

FIG. 4. $1 / N$ corrections to the $\psi$ field self-energy.

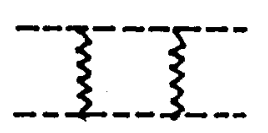

5. $a$

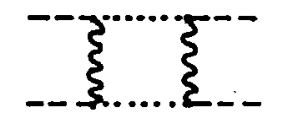

$5 . b$

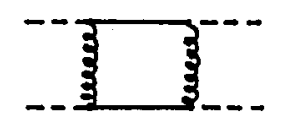

5.c

FIG. 5. Leading order contributions to the four point function of the scalar field $\varphi$.

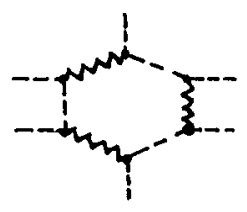

6. $a$

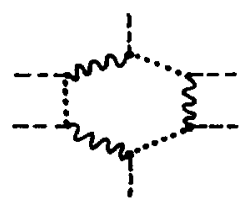

6.b

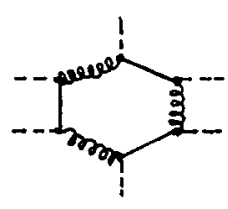

6.c

FIG. 6. Leading order contributions to the six point function of the scalar field $\varphi$.

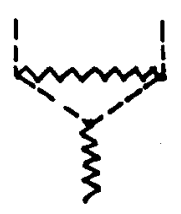

7.a

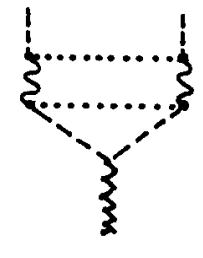

7.b

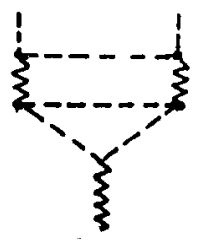

7.c

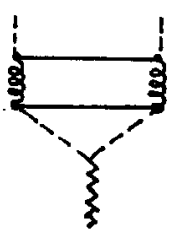

7.d

FIG. 7. Leading order contributions to the three point function $\Gamma_{\lambda \varphi \varphi}$. 